\title{
1 Opening the black box: interpretable machine learning for geneticists
}

2

3 Christina B. Azodi ${ }^{1,2,3 \uparrow}$, Jiliang Tang ${ }^{4}$, Shin-Han Shiu ${ }^{1,2,5 \uparrow}$

4

$5 \quad{ }^{1}$ Department of Plant Biology, Michigan State University, East Lansing, MI, USA

$6{ }^{2}$ The DOE Great Lakes Bioenergy Research Center, Michigan State University, East Lansing,

7 MI, USA

$8{ }^{3}$ Bioinformatics and Cellular Genomics, St. Vincent's Institute of Medical Research, Fitzroy,

9 Victoria, Australia

$10{ }^{4}$ Department of Computer Science and Engineering, Michigan State University, East Lansing,

11 MI, USA

$12{ }^{5}$ Department of Computational Mathematics, Science, and Engineering, Michigan State

13 University, East Lansing, MI, USA

14

15 Corresponding authors:

16 Christina B. Azodi

17 St. Vincent's Institute of Medical Research

189 Princes Street

19 Fitzroy, Victoria, 3065, Australia

20 Tel: +610433967476

21 E-mail: cazodi@svi.edu.au

22

23 Shin-Han Shiu

24 Michigan State University

25 Plant Biology Laboratories

$26 \quad 612$ Wilson Road, Room 166

27 East Lansing, MI 48824-1312, USA

28 Tel: $+1-517-353-7196$

29 E-mail: shius@msu.edu

30

31 Key words: interpretable machine learning, deep learning, predictive biology 


\section{Abstract}

33 Machine learning (ML) has emerged as a critical tool for making sense of the growing amount of

34 genetic and genomic data available because of its ability to find complex patterns in high

35 dimensional and heterogeneous data. While the complexity of ML models is what makes them

36 powerful, it also makes them difficult to interpret. Fortunately, recent efforts to develop

37 approaches that make the inner workings of ML models understandable to humans have

38 improved our ability to make novel biological insights using ML. Here we discuss the

39 importance of interpretable ML, different strategies for interpreting ML models, and examples of

40 how these strategies have been applied. Finally, we identify challenges and promising future

41 directions for interpretable ML in genetics and genomics.

\section{$43 \quad$ Highlights}

44 - Machine learning (ML) has emerged as a powerful tool for harnessing big biological 45 data.

46 - The complex structure underlying ML models means that their inner logic is not readily

47 intelligible to a human, hence the common critique of ML models as black boxes.

48 - However, advances in the field of interpretable ML have made it possible to identify

49 important patterns and features underlying a ML model using various strategies.

50 - These interpretation strategies have been successfully applied by researchers in genetics

51 and genomics to derive novel biological insights from ML models.

52 - This area of research is becoming increasingly important as more complex and difficult

53 to interpret ML approaches (i.e. deep learning) are being adopted by biologists. 


\section{Glossary}

56 Algorithm: The procedure taken to solve a problem/build a model.

57 Decision tree: A model made up of a series of branching true/false questions.

58 Deep Learning: A subset of ML algorithms inspired by the structure of the brain that can find 59 complex, nonlinear patterns in data.

60 Feature: An explanatory (i.e. independent) variable during modeling.

61 Global interpretation: A ML interpretation that explains the overall relationship between the 62 features and the label for all instances.

63 Instance: A single example from which the model will learn or be applied to.

64 Interpretable: Capable of being understood by a human.

65 Label: The variable to be predicted (i.e. the dependent variable).

66 Local interpretation: A ML interpretation that explains the relationship between the features

67 and the label for one or a subset of instances.

68 Machine learning: Computational models that learn from data without being explicitly 69 programmed.

70 Model: The set of patterns learned for a specific problem, where given input (i.e. instances and 71 their features) the model will generate an output (i.e. prediction).

72 Model performance: A quantitative evaluation of the model's ability to correctly predict labels.

73 Parameters: Variables in an ML model whose values are estimated/optimized during training.

74 Perturbing strategies: A family of interpretation strategies that measure how changes in the 75 input data impact model predictions or performance.

76 Probing strategies: A family of interpretation strategies that involve inspecting the structure and 77 parameters in a trained model.

78 Surrogate strategies: A family of interpretation strategies that involve training an inherently

79 interpretable model (e.g. a linear model) using the same data as a black-box model to serve as the 80 black-box model's surrogate.

81 Training: The process of identifying the best parameters to make up a model - the learning part 82 in ML. 


\section{Importance of interpretable machine learning}

84 Biological Big Data [1,2] has driven progresses in fields ranging from population genetics [3] to

85 precision medicine [4]. Much of this progress is possible because of advances in machine

86 learning (see Glossary; ML; Box 1) [5-10], "[a] field of study that gives computers the ability to

87 learn without being explicitly programmed" [11]. ML works by identifying patterns in data in the

88 form of a model that can be used to make predictions about new data. While powerful, ML also

89 presents new challenges. For example, a common criticism is that the ML models are "black

90 boxes", meaning their internal logic cannot be easily understood by a human [12]. Luckily,

91 strategies to demystify the inner working of ML models are available and ever improving.

92 There are three major reasons - troubleshooting, novel insights, and trust - why

interpretable ML model, or the ability to understand what logic is driving a model's prediction,

is important (Figure 1A, Key Figure). First, ML models rarely perform well without tweaking or

95 troubleshooting. Understanding how predictions are made is essential for identifying mistakes or biases in the input data and issues with how the model is trained. Second, an ML model with

97 impressive performance may have identified biologically novel patterns. However, such insights

98 will only be available if the model can be interpreted. Finally, we are unlikely to trust a

99 prediction if we do not understand why it was made. For example, a doctor may not trust a ML

100 diagnosis with no supporting justification out of concern that the model may be capturing

101 artifacts or have unknown biases or limitations [13].

Overview of strategies for interpretable machine learning

103 A wide range of strategies for interpretable ML have been developed and applied to problems in genetics and genomics [14-16]. These strategies can be characterized based on if they are applicable to all ML algorithms (i.e. model-agnostic) or only to one or a subset of algorithms

106 (i.e. model-specific). They can also be characterized based on if they provide global or local 107 interpretations. Global interpretations involve explaining the overall relationship between

108 features and labels. While local interpretations focus on explaining the prediction of an

109 individual instance. For example, imagine you train an ML model to predict if a gene (an 110 instance) is up-regulated after some treatment (the label) based on the presence or absence of a 111 set of regulatory sequences (the features). A global interpretation strategy will tell you how 
112 important regulatory sequence $\mathrm{X}$ is for predicting up-regulation across all genes in your dataset.

113 While a local interpretation strategy will tell you how important regulatory sequence $\mathrm{X}$ is for

114 predicting gene $\mathrm{Y}$ as up-regulated. This means that the type of interpretation strategy you select

115 will dictate what you will learn from your ML model, with different strategies possibly telling

116 different stories. We should also emphasize that ML models identify association through

117 correlation, thus ML interpretation strategies do not identify causal relationships between input

118 features and labels. Instead, interpretations should be used to generate new hypotheses that can

119 be tested experimentally. We will review three general ML interpretation strategies: probing,

120 perturbing, and surrogate strategies (Figure 1B; $[14,16]$ ).

121 Probing strategies dissect the inner structure of ML models

122 Training an ML model involves identifying the set of parameters best able to predict the label

123 of an instance (e.g. gene $\mathrm{Y}$ is up-regulated). After training, these parameters can be probed (or

124 inspected) to better understand what the model learned. Probing strategies provide global

125 interpretations with some exceptions (e.g. DeepLIFT, see below). Because type of parameters

126 and structure of how they connect to each other varies by algorithm, probing strategies are

127 model-specific. While probing strategies are straightforward for some ML algorithms (e.g.

128 Support Vector Machine; SVM; and decision tree-based algorithms), this is not the case for

129 more complex ML algorithms (e.g. deep learning).

130 Probing Support Vector Machine models

$131 \quad$ SVM is an algorithm that finds the hyperplane that best separates instances by their label

132 when they are plotted in $n$-dimensional space $(n=$ number of features). Training an SVM model

133 to predict gene up-regulation using regulatory sequences as features means learning the

134 combination of weights to apply to each regulatory sequence (i.e. coefficient weight) in order to

135 make the best hyperplane (Figure 2A). SVM models can be trained to learn either linear or non-

136 linear relationships between features and labels. While there are advanced methods for probing

137 non-linear SVM models [17,18], in most biological applications of SVM, only linear SVM

138 models are probed.

139 A trained linear SVM model is probed by extracting the coefficient weights that define

140 the hyperplane (Figure 2A), where features assigned a higher absolute weight have a stronger

141 relationship with the label and thus are more important for driving the prediction. For example, a 
142 linear SVM model was trained to classify simulated populations as being under positive or

143 negative selection using genetic markers as features [19]. Genetic markers with large, positive

144 coefficient weights in the SVM model were the same as those associated with positive selection

145 using classical population genetics statistical tests (e.g. Tajima’s $D$ ).

146 Importantly, SVM probing strategies (like other strategies discussed below), can provide

147 an incomplete picture of feature importance. For example, two highly correlated features will

148 split the weight between them, reducing their perceived importance. Or a feature with a strong

149 non-linear relationship with the label may not be assigned a large weight by a linear SVM model

150 and will therefore be missed when the trained model is probed.

\section{Probing decision tree-based models}

152 A decision tree is a set of true/false questions nested in a hierarchical structure. They are

153 inherently interpretable because the content and order of each question can be directly observed.

154 How well a true/false question separates instances by their label can also be quantified using

155 metrics such as the mean decrease in node impurity. In Figure 2B, using the presence/absence of

156 regulatory sequence "AACGT" to separate up- from down-regulated genes results in a decrease

157 in the mean node impurity. Because single decision trees tend to perform poorly at predicting

158 complex patterns, ensemble approaches (e.g. Random Forest, Gradient Tree Boosting [20]),

159 where many decision trees are combined to generate one prediction, are often used. Ensemble

160 decision-tree models can be probed by calculating the mean decrease in node impurity for each

161 feature across all trees in the ensemble. This approach was used determine which DNA motifs

162 were the most important for predicting if a gene would be differentially expressed under salt

163 stress conditions in Arabidopsis thaliana [21].

164 The hierarchical structure of decision tree-based models means that interactions between

165 features an be readily probed. For example, using a tool for finding stable feature interactions in

166 Random Forest models [21], Vervier and Michaelson identified interactions between genomic,

167 transcriptomic, and epigenomic features that were predictive of deleterious genetic variants [23].

168 Specifically, that an interaction between the local GC content and the distance to the nearest

169 expression Quantitative Trait Loci was important for predicting deleterious variants.

170 As with coefficient weights from SVM models, mean decrease impurity scores can be

171 misleading when features are highly correlated. This score also tends to inflate continuous over

172 categorical features, categorical features with a larger number of categories, and continuous 
173 features with a larger numeric range and should therefore be interpreted with caution when

174 feature space is not uniform [24].

\section{Probing deep learning networks}

176 While the classical ML algorithms described above are readily interpretable, deep

177 learning (Box 2) algorithms are being applied more and more in the ML community because

178 they frequently outperform classical ML algorithms at modeling complex systems [25-27] and

179 they can learn from raw data (e.g. whole DNA sequence) rather than user defined features (e.g.

180 known regulatory sequences). However, there is often a tradeoff between predictability and

181 interpretability [28], and this is certainly the case for deep learning [29]. Fortunately, there has

182 been a substantial effort to develop new methods to interpret these complex models. First we

183 describe three general approaches to calculate feature importance scores by probing deep

184 learning models: connection weights-based, gradient-based, and activation level-based

185 approaches (Figure 2C) [15].

186 Connection weight-based feature importance scores quantify the global relationship

187 between each feature and the output by summing the learned weights assigned to connections

188 between nodes in input-to-hidden, one or more hidden-to-hidden, and hidden-to-output layers for

189 each input feature [30,31]. Following the path through the example artificial neural network

190 (Figure 2C), the connection weights (represented by line widths) between some features (e.g. $\left.\mathrm{f}_{1}\right)$

191 and the output layer are larger than the connection weights between other features (e.g. $f_{3}$ ) and

192 the output layer, indicating $f_{1}$ is more important for that model. This approach was used to

193 determine which microRNA features were the most important for predicting the expression level

194 of Smad7, a gene involved in disrupting a signaling process up-regulated in patients with breast

195 cancer [32]. Connection weight-based feature importance scores can be misleading when feature

196 are on different scales, when positive and negative connection weights cancel each other out, or

197 when a connection has a large weight but is rarely activated (i.e. the nodes is rarely turned on)

198 [33].

199 The gradient-based feature importance scores (a.k.a. Saliency) also quantify the global

200 relationship between a feature and the output, but do so by calculating the gradient, or the change

201 in the predicted output (e.g. the likelihood a gene is up-regulated) as small changes are made to

202 the input feature (e.g. the frequency of regulatory sequence $\mathrm{X}$ ). The gradient is calculated using a

203 handy calculus trick, the partial derivative [34]. This approach was used to identify putative 
204 distal regulatory sequences in genomic regions where positive and negative gradient-based

205 importance score peaks represented enhancer and silencer regions, respectively [35]. This

206 approach is not useful when input features are categorical or when small changes in the feature

207 value do not change the output prediction [33].

208 Finally, the activation level refers to the output value from a node after it has passed 209 through a non-linear function (i.e. the activation function; see Box 2). Activation level-based

210 feature importance scores provide a local interpretation for an instance of interest by comparing

211 how much each feature activates nodes in the trained network compared to the feature values

212 from a reference instance. A reference instance for an image classification model could be one

213 that is solid white, while a reference for a model using a DNA sequence as instances could be an

214 instance with the background nucleotide frequency at every site. This approach (coined

215 DeepLIFT [33]), has been used in multiple biological studies [36-38]. For example, Zuallaert et

216 al. used DeepLIFT to find nucleotide sequences important for predicting splice sites [37].

217 Because DeepLIFT probes activation levels rather than connection weights, it avoids the pitfall

218 of the connection weight-based approach. Further, because it compares a specific instance to a 219 reference, it also avoids the pitfalls of the gradient-based approach.

220 Another way to probe deep learning models is to learn what pattern each node in the

221 network learned to identify (Figure 2C). This can be done by finding real or simulated instances

222 that maximally activate that node, then the properties of those real or simulated instances can be

223 used to interpret that node. For example, if the 10 DNA sequences that maximally activate node

$224 \mathrm{X}$ (i.e. cause node $\mathrm{X}$ to have the maximum possible output value after passing through the

225 activation function) all contain the motif ACGGTC, one could infer that node trained to find the

226 ACGGTC motif. Because probing every node in every layer may produce results that are still too

227 complex to interpret, dimensionality reduction techniques can be used to ease interpretation. For

228 example, Esteva et al. used a dimensionality reduction technique to visualize the nodes in the last

229 hidden layer of a convolutional neural network (see Box 2) trained to diagnose different types of

230 skin cancer from photos [39]. This allowed them to visualize how well their convolutional neural

231 network learned to separate different types of carcinomas. 


\section{Perturbing strategies for interpreting machine learning models}

233 Perturbing strategies involve modifying the input data and observing some change in the model

234 output. Because modifications to the input data can be made regardless of the ML algorithm

235 used, perturbing strategies are generally model-agnostic. We discuss two general perturbation-

236 based strategies: sensitivity analysis and what-if methods (Figure 3).

\section{Sensitivity Analysis}

Sensitivity analysis involves modifying an input feature and measuring the impact on model performance (Figure 3A). Feature modification typically means removing (i.e. leaveone-feature-out) or permuting (e.g. set all values to the mean) one feature at a time. The decrease

242 in model performance after a feature is removed or permuted is an intuitive score for each feature

243 indicating its contribution to the predictions (Figure 3A). Because perturbing a feature not only

244 impacts that feature but also other features that interact with it, sensitivity analysis also captures

245 interaction effects for each feature. However, sensitivity analysis can miss important features if

246 correlation exists in the feature set. For example, if features X and Y are highly correlated,

247 feature $\mathrm{Y}$ could compensate when $\mathrm{X}$ is removed or permuted, masking its potential importance.

248 Che et al. used the leave-one-feature-out approach to find that genomic region length was

249 the most important feature for identifying genomic regions that contain clusters of genes

250 acquired by horizontal gene transfer [40]. Leave-one-feature-out analysis is computationally

251 expensive because it requires training a new model for every perturbed dataset. Therefore, it is

252 typically not used to interpret deep learning model (which are already computing intensive)

253 except when there are few input features. For example, leave-one-feature-out was used to

254 determine that, of five histone marks, removing H3K4me3 resulted in the largest decrease in a

255 deep learning model's ability to predict TF binding sites [41].

256 Permutation strategies determine feature importance score by measuring how the

257 performance of an ML model changes when different features are randomly permuted. They are

258 more computationally efficient than leave-one-feature-out strategies because only one model

259 needs to be trained. This strategy is particularly intriguing for genetic studies because its logic is

260 similar to DNA mutagenesis experiments. It was demonstrated that in silico mutagenesis (i.e.

261 computationally permuting DNA sequence) could identify which nucleotides impact tissue 
262 specific gene expression the most [42]. A permutation-based strategy used in image analysis is

263 called occlusion sensitivity. Here different regions in images are grayed out and the resulting

264 change in performance is measured. For example, occlusion of regions of blood smear images

265 confirmed that a malaria classification model performed worst when parasitized regions were

266 grayed out [43].

267

268 What-if Analysis

269 The what-if approach (a.k.a. counterfactuals [44]) measures how the prediction of a

270 particular instance changes (rather than the overall model performance) when the input value for

271 one or more features is changed. Thus, what-if analysis provides local interpretations while

272 sensitivity analysis provides global interpretations. Here we focus on two what-if methods:

273 partial dependency plots (PDPs) and individual conditional expectation (ICE) plots (Figure 3B;

$274[16])$.

275 PDPs show how a prediction changes when the input value for a feature of interest is

276 changed, marginalizing (i.e. ignoring) the effects of all other features [45]. Imagine we trained a

277 ML model that predicts the likelihood that a sequence will be bound by a certain transcription

278 factor (TF). A PDP would show, for example, how the TF-binding likelihood would change if

279 the nucleotide at position of interest is changed from C to A, G or T (left panel, Figure 3B). This

280 approach was used to demonstrate the impact of sequence features (e.g. amino acid identity,

281 conservation) on the predicted efficacy of a guide RNA for CRISPR-Cas9 [46]. PDPs can miss

282 important features when there are interactions between features. For example, imagine if a $\mathrm{C}$ at

283 position \#3 increased TF binding affinity when position \#2 contained a $\mathrm{T}$ but decreased binding

284 affinity if position \#2 contained an A. Because position \#2 is marginalized in the position \#3's

285 PDP, the interaction may mask the importance of position \#3.

286 ICE plots were proposed to address this limitation of PDPs [47]. ICE plots are essentially

287 PDPs generated for every individual instance in the dataset. For example, an ICE plot for

288 position \#3 would show that the presence of a $\mathrm{C}$ at position \#3 only increases the TF binding

289 likelihood in the subset of sequences, which with further investigation we find are the sequences

290 with a T in position \#2 (right panel, Figure 3B). Because this strategy does not require model re-

291 training, it is well suited for interpreting deep learning models. For example, ICE plots were used

292 to better understand what patterns of gene expression an adversarial deep learning model (see 
293 Box 2, Figure IIB) learned were characteristic of single cell data [48]. By varying the expression

294 level of individual genes (the feature) within the single cell (the instance), they found the genes

295 with the biggest impact on the prediction (real or not) were genes known to be markers for

296 particular cell-type states (e.g. IvI, Krt10, and Krt14 for epidermal cell state).

297 What-if analyses can provide highly detailed and intuitive interpretations of ML models,

298 including the magnitude, direction, and non-linearities in the relationships between features and

299 the output label. A limitation is that PDP and ICE plots can only be visualized for one or two

300 features at a time, so they are typically only generated for models with few features or with a

301 subset of features deemed important by another interpretation strategy or from domain

302 knowledge [49].

303 Surrogate strategies for interpreting machine learning models

304 Image you have an ML model that is truly a black box - meaning that it cannot be probed and

305 perturbations strategies do not provide useful information. In such a case, one can train an

306 inherently interpretable model (e.g. linear model or a decision tree) to act as a surrogate for the

307 black box model. For example, to generate a surrogate model for a black box model that can

308 predict gene up-regulation using regulatory elements as features, we would first apply the black

309 box model to a set of genes, $G$, and extract the black box predicted label (i.e. up- or down-

310 regulated) for those genes (Figure 1B). Then we would use the same set of genes $G$ as the

311 instances and the black box predicted labels as the labels to train an interpretable surrogate

312 model.

313 One major limitation of surrogate models is that black box models are often highly

314 complex (e.g. highly non-linear, many higher order interactions), and thus, cannot be fully

315 learned by an interpretable surrogate. To overcome this, one approach is to generate a surrogate

316 to learn just a portion of the black box model, known as a Local Interpretable Model-agnostic

317 Explanations (LIME; [50]). While the complex logic underlying the whole model may be too

318 much for a surrogate model to learn, the logic for one instance or a group of similar instances

319 (e.g. co-expressed genes) may be simple enough. For example, LIME was used to better

320 understand why some patients (i.e. instances) were misclassified by a black box model predicting

321 survival after cardiac arrest [51]. A LIME model for a patient that was mis-predicted to survive

322 showed that the black box model was too heavily influenced by certain features (e.g. healthy 
323 neurologic status, lack of chronic respiratory illness) and did not place sufficient weight on other

324 features that are also important (e.g. elevated creatinine, advanced age).

\section{Concluding Remarks}

326 Interpretability is critical for applications of ML in genetics and beyond and will therefore see

327 substantial advances in the coming years. Just as there is no one universally best ML algorithm,

328 there will not likely be one ML interpretation strategy that works best on all data or for all

329 questions. Rather, the interpretation strategy should be tailored to what you want to learn from

330 the ML model and confidence in the interpretation will come when multiple approaches tell the

331 same story. Luckily, many user-friendly tools have already been developed to facilitate

332 interpreting ML models using the strategies described in this review and more (Table 1). The

333 insights that can be learned from interpreting a ML model are constrained by the content, quality,

334 and quantity of the data used to generate the model. Care should be taken when selecting data

335 and features to avoid introducing technical or biological artifacts into the models, and thus into

336 the interpretations.

337 There are still many challenges to interpreting machine learning models in genetics and

338 genomics (see Outstanding Questions). These challenges, while not necessarily unique to

339 genetics or genomics, represent opportunities for computational biologists to innovate and

340 contribute novel solutions. They also highlight the importance of training the next generation of

341 biologists able to work at the intersection of computer and biological science.

\section{Acknowledgement}

343 We thank Yuying Xie for his insightful feedback on this review. This work was partly supported

344 by the National Science Foundation (NSF) Graduate Research Fellowship (Fellow ID:

$3452015196719)$ to C.B.A.; NSF (IIS-1907704, IIS-1845081, CNS- 1815636) grants to J.T.; and the

346 U.S. Department of Energy Great Lakes Bioenergy Research Center (BER DE-SC0018409) and

347 NSF (IOS-1546617, DEB-1655386) grants to S.-H.S. 
348

349

350

351

352

353

354

355

356

357

358

359

360

361

362

363

364

365

366

367

368

369

370

371

372

\section{Text Boxes}

374

375

376

\section{Outstanding Questions}

- How can we interpret ML models trained on heterogeneous (e.g. multi-omic) and high dimensional (number of features $>>$ number of instances) data? ML algorithms are well suited to take advantage of the large-scale multi-omic data for generating predictive models. However, interpreting ML models trained on high dimensional and heterogenous data remains challenging. These challenges are exasperated when features are highly correlated and of different types (e.g. continuous verses binary).

- What ML modeling and interpretation strategies are best for studying complex biological systems? Given the importance of non-linear effects in biology (e.g. epistasis, feedback loops, community dynamics, synergistic/antagonistic effects), interpretation strategies that can identify features that have important but complex effects are critical.

- How can we compare ML interpretation strategies and results? The strategies used to interpret an ML model are able to identify different aspects of the logic underlying that model. How can we benchmark new and established interpretation strategies for applications in genetics and genomics? Further, how could we join the findings from multiple strategies into a fuller, yet still coherent, interpretation of that model?

- How can interpretable ML become an accessible tool for biologists? Implementing ML interpretation strategies can require extensive computational knowledge. What roles will interdisciplinary training (e.g. computer science, data science) and the user-friendlysoftware play in encouraging the interpretation of ML models in genetics and genomics?

- How can researchers ensure that model interpretability will continue to be an area of development for folks working in the artificial intelligence field? As the power and precision of ML models improves, more and more trust will likely be placed in them. What role can researchers play in shaping the future of AI? 
377 is composed of instances (i.e. samples) and feature (i.e. independent variables) that describe 378 those instances. For example, if our instances are genes, features describing those genes could be 379 the GC content, the presence or absence of a specific functional domain, or its level of

380 conservation across species. If the values being predicted are not known a priori for any instance, 381 then unsupervised ML approaches (e.g. clustering) can be applied to extract previously unknown 382 patterns. If the values being predicted are known for some of the instances, these values are 383 referred to as labels and one can learn from these labels and turn the problem into a supervised 384 ML problem. Further, if the known labels are categorical (e.g. is the gene up-regulated or down385 regulated), it is a classification problem, while if the labels are continuous (e.g. gene expression 386 levels), it is a regression problem.

A common supervised ML workflow involves four steps: training, applying, scoring, and interpretation (Figure I). First, input data made up of features and labels for many instances are divided into a training set and a testing set. The features and labels from the training set are then used to train the ML model. During training, the ML model learns the combination of internal parameters that minimize the error in the predictions of the labels. Second, the trained ML model is applied to the testing set features to generate predicted labels. A trained ML model can also be applied to unlabeled instances to make predictions. Third, the performance of the ML models is scored by comparing the predicted labels with the known labels from the test set. Many different performance metrics are used in the ML field, where the best metric depends on the type of ML problem and the nature of the question being asked. A performance metric not only informs the quality of a model, but also provides a quantitative measure of how much we known about the biological phenomenon in question given the features used. Finally, the ML model is interpreted to provide a better, quantitative understanding on how the input features contribute to the predictions.

Figure I. A supervised machine learning workflow.

Box 2: A crash course in deep learning.

ML algorithms inspired by the structure of the brain make up a subfield of ML called Deep Learning (DL). DL is promising for biology because DL models can 1) learn highly complex nonlinear patterns, 2) continue to improve when given more training data ("shallow" 
408 ML models tend to plateau), and 3) they can learn from raw data without user defined features

409 [52]. A DL model is made up of multiple layers of nodes connected by edges of different

410 connection weights $\left(w_{\mathrm{x}}\right)$ (Figure IIA). The nodes in the input layer contain the feature values $\left(f_{x}\right)$

411 for an instance. The nodes in the hidden layers (hidden nodes) represent the sum of the nodes

412 from the previous layer multiplied by their associated connection weights $\left(\sum w_{x} f_{x}\right)$. The node

413 value from that summation is then passed through an activation function (represented as a light

414 switch), which determines the extent to which that node gets turned on (i.e. activated). A DL

415 models are able to learn nonlinear relationships when the activation function used is nonlinear

416 (e.g. the sigmoid function). The output node (i.e. the predicted label) is the sum of the nodes

417 from the last hidden layer and can be compared to the true label to calculate the error in the

418 model. A DL model is trained by propagating that error back through the model and updating the

419 learned connection weights (i.e. backpropagation of the error) until that error is minimized.

420 While this type of DL algorithm, often referred to as a fully-connected artificial neural

421 network, is useful for modeling complex, nonlinear relationships. Other DL algorithms many be

422 useful for addressing different biological questions (Figure IIB). For example, convolutional

423 neural networks learn spatial patterns making them ideal for identifying sequence motifs and

424 patterns in images, while recurrent neural networks remember earlier predictions and are

425 therefore ideal for sequential data analysis.

426

427 Figure II. Graphical explanations of deep learning algorithms. (A) An example fully-

428 connected artificial neural network. (B) Uses, graphical explanations, and example biological

429 applications for three additional deep learning algorithms: Convolutional Neural Networks,

430 Recurrent Neural Networks, and Adversarial Learning.

431

$432 \quad$ Figure Legends

433 Figure 1. Overview of ML model interpretation strategies

434 (A) Understanding the inner logic of a machine learning (ML) model (i.e. model interpretability),

435 is important for troubleshooting during model training, generating biological insights, and

436 instilling trust in the predictions made. (B) There are three general strategies for interpreting a

437 ML model: probing, perturbing, and surrogates. Probing strategies involve inspecting the 
438 structure and parameters learned by a trained ML model (e.g. a deep learning model pictured

439 here) in order to better understand what features or combination of features are important for 440 driving the model's predictions. Perturbing strategies involve changing values of one or more

441 input features (e.g. setting all values to zero) and measuring the change in model performance

442 (sensitivity analysis) or on the predicted label of a specific instance (what if analysis). Finally, an

443 easily interpretable model (e.g. linear regression or decision tree) can be trained to predict the

444 predictions from a ML models, acting as a surrogate.

Figure 2. Probing a trained machine learning model.

447 An ML model that classifies up- (green) from down-regulated (yellow) genes using regulatory

448 sequences (purple) as features can be probed to find what regulatory sequences are most

449 important for predicting differential expression. (A) A support vector machine model learns the

450 combination of coefficient weights ( $w$; orange) that form the decision boundary (dotted line) best

451 able to separate up- from down-regulated genes, where the features assigned the higher $w$ are

452 more important. The decision boundary is a hyperplane represented by the equation shown. (B)

453 A decision tree-based model learns the most predictive series of true/false questions about the

454 features. Here we zoom in on a node where the regulatory sequence "AACGT" is used as the

455 feature. How well AACGT separates up- from down-regulated genes is quantified by calculating

456 the mean decrease in node impurity after AACGT is used. Large impurity scores (here calculated

457 as the Gini Impurity) mean the node contains a mix of up and down-regulated genes, while an

458 impurity score equal to zero would indicate the node only contains up or down-regulated genes.

459 (C) Deep learning models train to learn what combinations of connection weights (gray lines)

460 across all nodes and layers results in the network best able to classify up- from down-regulated

461 genes. A trained deep learning models can be probed by inspecting the size of the connection

462 weights (gray line thickness), measuring the gradient of the output with respect to the input [i.e.

$463 \partial \operatorname{Out}(\mathrm{in}) / \partial$ (in)], and quantifying the extent to which different features cause a node to activate

464 (represented by the light switch).

466 Figure 3. Perturbing the input to a machine learning model.

467 An example ML model predicting if a Transcriptional Factor (TF) may bind (i.e. the label) to a 468 specific sequence (i.e. the features) can be interpreted with perturbing strategies. (A) Sensitivity 
469 analysis. Leave-one-feature-out means a new ML model is trained on the same input data with

470 one feature (e.g. position 3) removed. Then the overall performance of the original model and the

471 new model are compared. Permutation means the original model is applied to input data with the

472 values shuffled for one feature at a time. The performance of the model applied to the original

473 and the shuffled data are compared. Both sensitivity analyses on position 3 shown here resulted

474 in a decrease in performance, leading to the interpretation that position 3 is important for TF

475 binding. (B) What-If analysis. The partial dependency plot (left) shows the TF binding likelihood

476 if position 3 was an A, C, G, or T, ignoring the effects of nucleotides at other positions. This plot

477 shows that a $\mathrm{C}$ at position 3 increases the likelihood of $\mathrm{TF}$ binding. The individual conditional

478 expectation plot (right) shows the TF binding likelihood score for every instance (dot) in the

479 dataset when position 3 is A, C, G, or T. This plot shows when position 3 is C, the binding

480 likelihoods have a bimodal distribution which is due to interaction with position 2 in this

481 hypothetical example.

482

483

Table Legends

484 Table 1. Platforms and software available for interpretable machine learning

\begin{tabular}{|c|c|c|c|c|c|}
\hline Name & Strategy & Use & Scope & Description & Platform \\
\hline $\begin{array}{l}\text { CamurW } \\
\text { eb [53] }\end{array}$ & Probing & $\begin{array}{l}\text { Decision } \\
\text { tree-based } \\
\text { models }\end{array}$ & Global & $\begin{array}{l}\text { Interpret decision rules } \\
\text { from Classifier with } \\
\text { Alternative and } \\
\text { MUltiple Rule (Camur) } \\
\text { models }\end{array}$ & web tool \\
\hline $\begin{array}{l}\text { DeepExp } \\
\text { lain [54] }\end{array}$ & $\begin{array}{l}\text { Probing, } \\
\text { perturbing }\end{array}$ & $\begin{array}{l}\text { Deep } \\
\text { Learning }\end{array}$ & $\begin{array}{l}\text { Global, } \\
\text { local }\end{array}$ & $\begin{array}{l}\text { Toolbox for } \\
\text { implementing multiple } \\
\text { interpretation methods }\end{array}$ & $\begin{array}{l}\text { Tensorflow, } \\
\text { Keras }\end{array}$ \\
\hline $\begin{array}{l}\text { Deep TRI } \\
\text { AGE } \\
{[55]}\end{array}$ & Probing & $\begin{array}{l}\text { Attention- } \\
\text { based } \\
\text { Deep } \\
\text { Learning }\end{array}$ & Local & $\begin{array}{l}\text { Deep learning for the } \\
\text { Tractable } \\
\text { Individualized Analysis } \\
\text { of Gene Expression }\end{array}$ & $\begin{array}{l}\text { Python } \\
\text { package }\end{array}$ \\
\hline
\end{tabular}




\begin{tabular}{|c|c|c|c|c|c|}
\hline $\begin{array}{l}\text { iml: } \\
\text { interpreta } \\
\text { ble ML } \\
{[16]}\end{array}$ & $\begin{array}{l}\text { Probing, } \\
\text { perturbing }\end{array}$ & $\begin{array}{l}\text { Model } \\
\text { agnostic }\end{array}$ & $\begin{array}{l}\text { Global, } \\
\text { Local }\end{array}$ & $\begin{array}{l}\text { Toolbox for } \\
\text { implementing multiple } \\
\text { interpretation methods. }\end{array}$ & R package \\
\hline $\begin{array}{l}\text { iNNvesti } \\
\text { gate [56] }\end{array}$ & Probing & $\begin{array}{l}\text { Deep } \\
\text { Learning }\end{array}$ & $\begin{array}{l}\text { Global, } \\
\text { Local }\end{array}$ & $\begin{array}{l}\text { Toolbox for } \\
\text { implementing multiple } \\
\text { interpretation methods. }\end{array}$ & Keras \\
\hline iRF [22] & Probing & $\begin{array}{l}\text { Random } \\
\text { Forest }\end{array}$ & Global & $\begin{array}{l}\text { Decision tree based } \\
\text { method to identify } \\
\text { significant feature } \\
\text { interactions. }\end{array}$ & R package \\
\hline $\begin{array}{l}\text { LIME } \\
{[50]}\end{array}$ & Surrogate & $\begin{array}{l}\text { Model } \\
\text { Agnostic }\end{array}$ & Local & $\begin{array}{l}\text { A tool to generate local } \\
\text { surrogate models for } \\
\text { Black-Box models. }\end{array}$ & $\begin{array}{l}\text { Python } \\
\text { package }\end{array}$ \\
\hline $\begin{array}{l}\text { Lucid } \\
\text { (github.c } \\
\text { om/tenso } \\
\text { rflow/luci } \\
\text { d) }\end{array}$ & Probing & $\begin{array}{l}\text { Deep } \\
\text { Learning }\end{array}$ & $\begin{array}{l}\text { Global, } \\
\text { local }\end{array}$ & $\begin{array}{l}\text { Toolbox of methods for } \\
\text { visualizing and } \\
\text { interpreting neural } \\
\text { networks. }\end{array}$ & Tensorflow \\
\hline $\begin{array}{l}\text { NeuralNe } \\
\text { tTools } \\
{[57]}\end{array}$ & $\begin{array}{l}\text { Probing, } \\
\text { perturbing }\end{array}$ & $\begin{array}{l}\text { Deep } \\
\text { Learning }\end{array}$ & $\begin{array}{l}\text { Global, } \\
\text { local }\end{array}$ & $\begin{array}{l}\text { Toolbox for } \\
\text { implementing multiple } \\
\text { interpretation methods. }\end{array}$ & R package \\
\hline $\begin{array}{l}\text { SpliceRo } \\
\text { ver [37] }\end{array}$ & Probing & $\begin{array}{l}\text { Deep } \\
\text { Learning }\end{array}$ & Local & $\begin{array}{l}\text { Tool to interpret which } \\
\text { nucleotides contribute } \\
\text { most predicting splice } \\
\text { sites using DeepLIFT }\end{array}$ & web tool \\
\hline $\begin{array}{l}\text { The } \\
\text { What-If } \\
\text { Tool } \\
\text { (https://p } \\
\text { air- }\end{array}$ & $\begin{array}{l}\text { Probing, } \\
\text { perturbing }\end{array}$ & $\begin{array}{l}\text { Model } \\
\text { Agnostic }\end{array}$ & $\begin{array}{l}\text { Global, } \\
\text { local }\end{array}$ & $\begin{array}{l}\text { Code free toolbox for } \\
\text { assessing, comparing, } \\
\text { and interpreting } \\
\text { Tensorflow/python- } \\
\text { based ML models }\end{array}$ & $\begin{array}{l}\text { TensorBoard, } \\
\text { Jupyter, } \\
\text { Colaboratory } \\
\text { notebooks }\end{array}$ \\
\hline
\end{tabular}




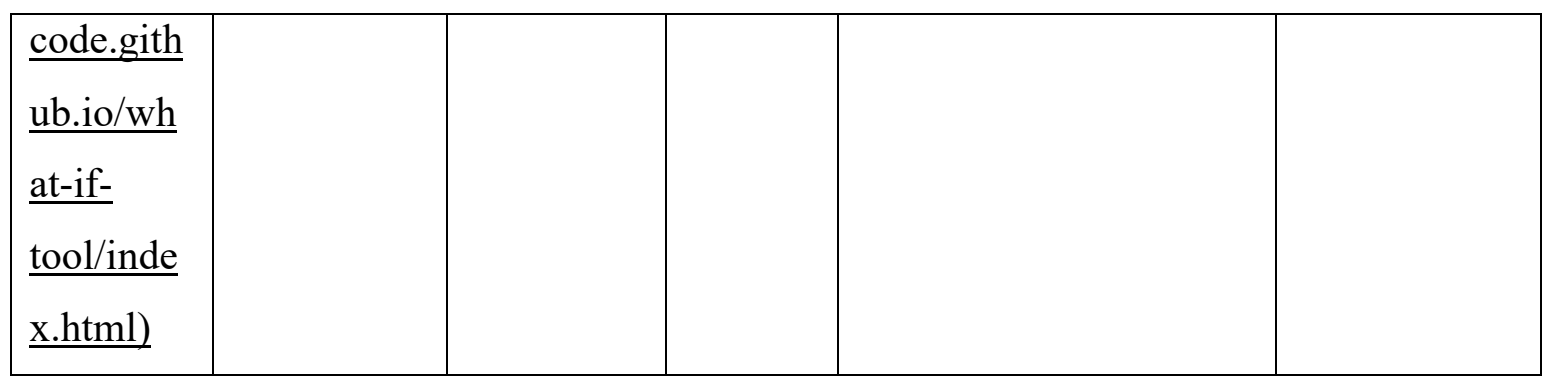

485

486 References

4871 Marx, V. (2013) Biology: The big challenges of big data. Nature 498, 255-260

4882 Stephens, Z.D. et al. (2015) Big Data: Astronomical or Genomical? PLOS Biol. 13, e1002195

4893 Schrider, D.R. and Kern, A.D. (2018) Supervised Machine Learning for Population Genetics:

490 A New Paradigm. Trends Genet. 34, 301-312

4914 Alyass, A. et al. (2015) From big data analysis to personalized medicine for all: challenges

492 and opportunities. BMC Med. Genomics 8, 33

4935 Angermueller, C. et al. (2016) Deep learning for computational biology. Mol. Syst. Biol. 12,

$494 \quad 878-16$

4956 Chicco, D. (2017) Ten quick tips for machine learning in computational biology. BioData

496 Min. 10, 35

4977 Cuperlovic-Culf, M. (2018) Machine Learning Methods for Analysis of Metabolic Data and

498 Metabolic Pathway Modeling. Metabolites 8, 4

4998 Libbrecht, M.W. and Noble, W.S. (2015) Machine learning applications in genetics and

500 genomics. Nat. Publ. Group 16, 321-332

5019 Ma, C. et al. (2014) Machine learning for Big Data analytics in plants. Trends Plant Sci.

50210 Tarca, A.L. et al. (2007) Machine Learning and Its Applications to Biology. PLOS Comput.

503 Biol. 3, e116

504

505

506

507

508

509

510

11 Samuel, A.L. (1959) Some Studies in Machine Learning Using the Game of Checkers. IBM J. Res. Dev. 3, 210-229

12 Lipton, Z.C. (2018) The Mythos of Model Interpretability. ACM Queue 16,

13 Miller, T. (2019) Explanation in artificial intelligence: Insights from the social sciences. Artif. Intell. 267, 1-38

14 Guidotti, R. et al. (2018) A Survey of Methods for Explaining Black Box Models. ACM Comput. Surv. 51, 1-42

15 Montavon, G. et al. (2018) Methods for interpreting and understanding deep neural networks. Digit. Signal Process. 73, 1-15

16 Molnar, C. (2019) Interpretable Machine Learning: A Guide for Making Black Box Models Explainable, 1st edition.Christoph Molnar.

17 Barakat, N. and Bradley, A.P. (2010) Rule extraction from support vector machines: A review. Neurocomputing 74, 178-190

18 Rasmussen, P.M. et al. (2011) Visualization of nonlinear kernel models in neuroimaging by 518 sensitivity maps. Neurolmage 55, 1120-1131 
19 Ronen, R. et al. (2013) Learning Natural Selection from the Site Frequency Spectrum. Genetics 195, 181-193

20 Breiman, L. (2001) Random Forests. Mach. Learn. 45, 5-32

21 Uygun, S. et al. (2019) Cis-regulatory code for predicting plant cell-type transcriptional response to high salinity. Plant Physiol. DOI: 10.1104/pp.19.00653

22 Basu, S. et al. (2018) Iterative random forests to discover predictive and stable high-order interactions. Proc. Natl. Acad. Sci. 115, 1943-1948

23 Vervier, K. and Michaelson, J.J. (2018) TiSAn: estimating tissue-specific effects of coding and non-coding variants. Bioinformatics 34, 3061-3068

24 Strobl, C. et al. (2007) Bias in random forest variable importance measures: Illustrations, sources and a solution. BMC Bioinformatics 8,

25 Banerjee, S. et al. (2017) , Performance of Deep Learning Algorithms vs. Shallow Models, in Extreme Conditions - Some Empirical Studies. , in Pattern Recognition and Machine Intelligence, pp. 565-574

26 Guo, Y. et al. (2016) Deep learning for visual understanding: A review. Neurocomputing 187, 27-48

27 LeCun, Y. et al. (2015) Deep learning. Nature 521, 436-444

28 Kuhn, M. and Johnson, K. (2013) Applied Predictive Modeling,

29 Schmidhuber, J. (2015) Deep learning in neural networks: An overview. Neural Netw. 61, 85-117

30 Garson, D.G. (1991) Interpreting neural network connection weights. Al Expert 6, 46-51

31 Olden, J.D. and Jackson, D.A. (2002) Illuminating the "black box": a randomization approach for understanding variable contributions in artificial neural networks. Ecol. Model. 154, 135-150

32 Manzanarez-Ozuna, E. et al. (2018) Model based on GA and DNN for prediction of mRNASmad7 expression regulated by miRNAs in breast cancer. Theor. Biol. Med. Model. 15,

33 Shrikumar, A. et al. (2017) Learning Important Features Through Propagating Activation Differences. Proc. 34 Th Int. Conf. Mach. Learn. at <http://proceedings.mlr.press/v70/shrikumar17a/shrikumar17a.pdf>

34 Simonyan, K. et al. (2013) Deep Inside Convolutional Networks: Visualising Image Classification Models and Saliency Maps. Int. Conf. Learn. Represent. at <http://arxiv.org/abs/1312.6034>

35 Kelley, D.R. et al. (2018) Sequential regulatory activity prediction across chromosomes with convolutional neural networks. Genome Res. 28, 739-750

36 Washburn, J.D. et al. (2019) Evolutionarily informed deep learning methods for predicting relative transcript abundance from DNA sequence. Proc. Natl. Acad. Sci. 116, 5542-5549

37 Zuallaert, J. et al. (2018) SpliceRover: interpretable convolutional neural networks for improved splice site prediction. Bioinformatics 34, 4180-4188

38 Kim, J.-S. et al. (2018) RIDDLE: Race and ethnicity Imputation from Disease history with Deep LEarning. PLOS Comput. Biol. 14, e1006106

39 Esteva, A. et al. (2017) Dermatologist-level classification of skin cancer with deep neural networks. Nature 542, 115-118

40 Che, D. et al. (2010) Classification of genomic islands using decision trees and their ensemble algorithms. BMC Genomics 11, S1 
56341 Jing, F. et al. (2019) An integrative framework for combining sequence and epigenomic data Biol. Bioinform. DOI: 10.1109/TCBB.2019.2901789

42 Zhou, J. et al. (2018) Deep learning sequence-based ab initio prediction of variant effects on expression and disease risk. Nat. Genet. 50, 1171-1179

43 Rajaraman, S. et al. (2018) Understanding the learned behavior of customized convolutional neural networks toward malaria parasite detection in thin blood smear images. J. Med. Imaging 5, 1

44 Wachter, S. et al. (2018) Counterfactual explanations without opening the black box: automated decisions and the GDPR. Harv. J. Law Technol. 31,

45 Friedman, J.H. (2001) Greedy function approximation: A gradient boosting machine. Ann. Stat. 29, 1189-1232

46 Schoonenberg, V.A.C. et al. (2018) CRISPRO: identification of functional protein coding sequences based on genome editing dense mutagenesis. Genome Biol. 19, 169

47 Goldstein, A. et al. (2015) Peeking Inside the Black Box: Visualizing Statistical Learning With Plots of Individual Conditional Expectation. J. Comput. Graph. Stat. 24, 44-65

48 Ghahramani, A. et al. (2018) Generative adversarial networks simulate gene expression and predict perturbations in single cells. bioRxiv DOI: $10.1101 / 262501$

49 Liu, Z. and Yang, J. (2014) Quantifying ecological drivers of ecosystem productivity of the early-successional boreal Larix gmelinii forest. Ecosphere 5, art84

50 Ribeiro, M.T. et al. (2016) , "Why Should I Trust You?": Explaining the Predictions of Any Classifier. , in Proceedings of the 22nd ACM SIGKDD International Conference on Knowledge Discovery and Data Mining - KDD '16, San Francisco, California, USA, pp. 1135-1144

51 Nanayakkara, S. et al. (2018) Characterising risk of in-hospital mortality following cardiac arrest using machine learning: A retrospective international registry study. PLOS Med. 15, e1002709

52 Eraslan, G. et al. (2019) Deep learning: new computational modelling techniques for genomics. Nat. Rev. Genet. 20, 389-403

53 Weitschek, E. et al. (2018) CamurWeb: a classification software and a large knowledge base for gene expression data of cancer. BMC Bioinformatics 19, 354

54 Ancona, M. et al. (2018) Towards better understanding of gradient-based attribution methods for Deep Neural Networks. ArXiv171106104 Cs Stat at $<$ http://arxiv.org/abs/1711.06104>

55 Beykikhoshk, A. et al. (2019) DeepTRIAGE: Interpretable and Individualised Biomarker Scores using Attention Mechanism for the Classification of Breast Cancer Sub-types. bioRxiv DOI: 10.1101/533406

56 Alber, M. et al. (2018) iNNvestigate neural networks! ArXiv180804260 Cs Stat at <http://arxiv.org/abs/1808.04260>

57 Beck, M.W. (2018) NeuralNetTools: Visualization and Analysis Tools for Neural Networks. J. Stat. Softw. 85, 1-20 
Figure 1

A

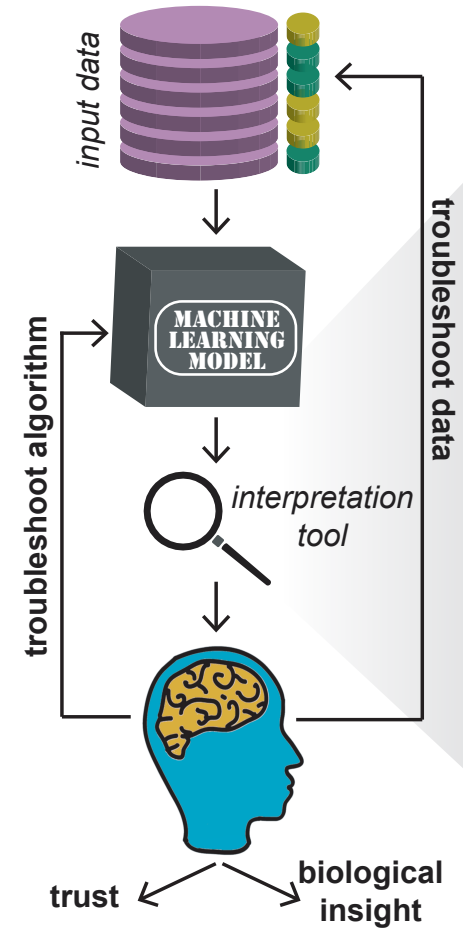

B

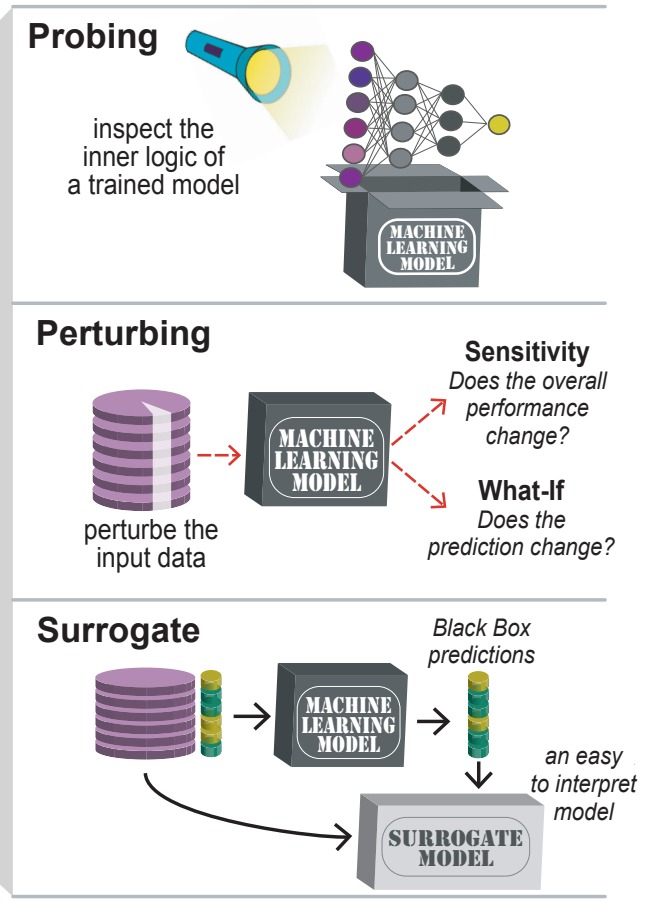




\section{Figure 2}

A

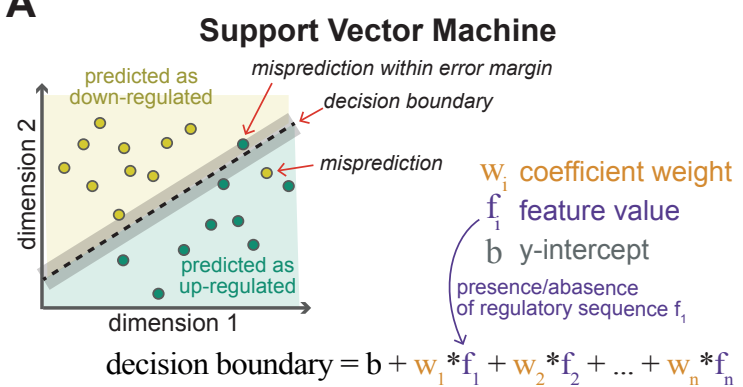

B
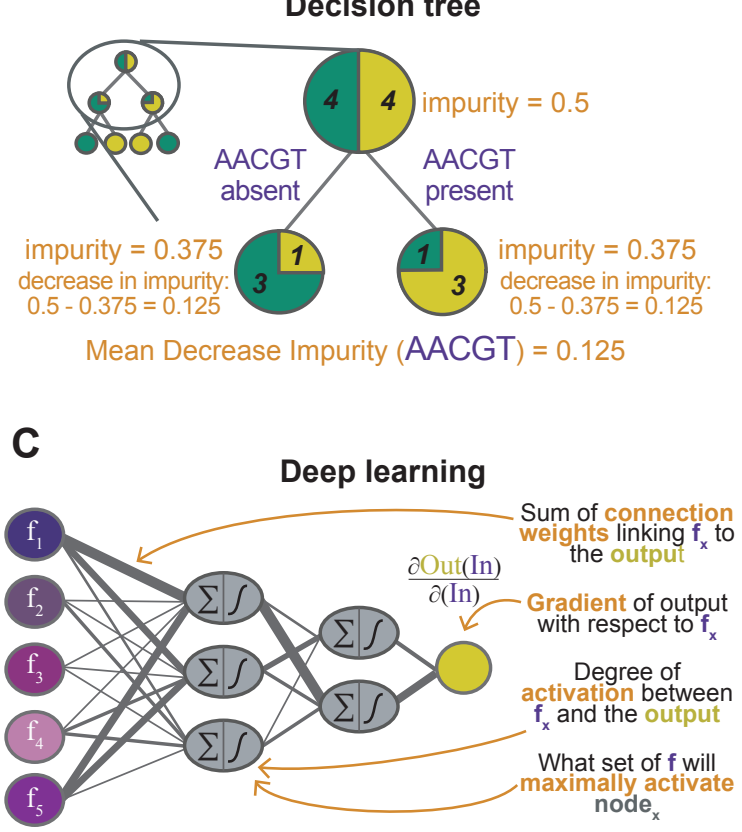
Figure 3

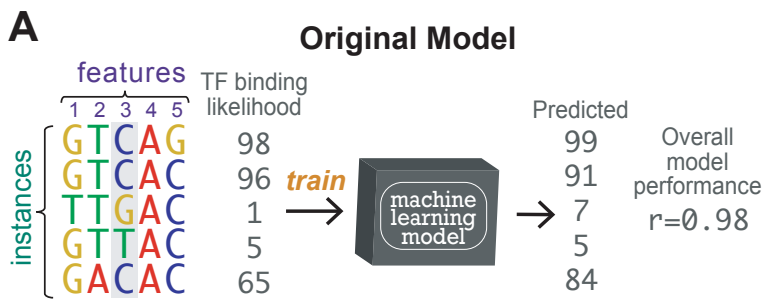

Leave-One-Feature-Out Sensitivity

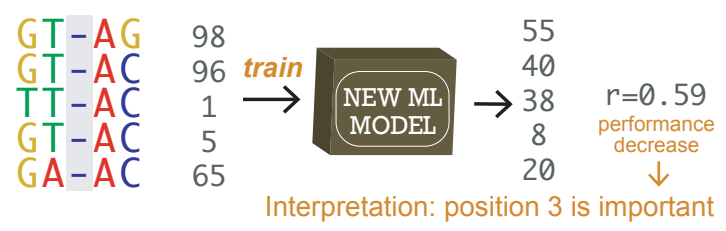

Permutation Sensitivity

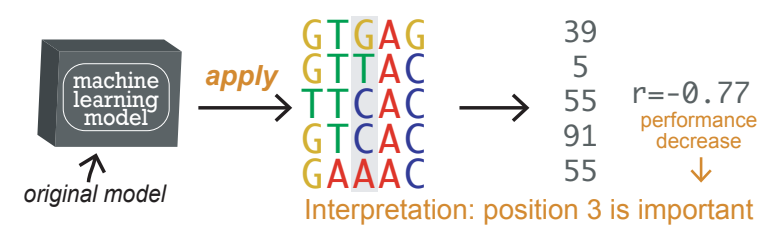

B



\section{Figure I}






\section{Figure II}

A

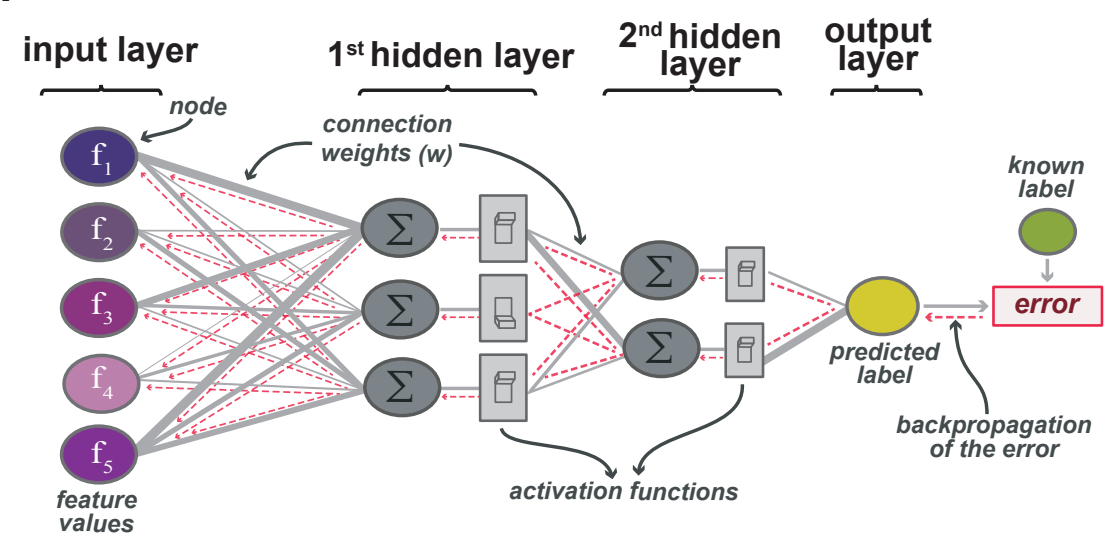

\section{B Convolutional}

$\stackrel{\oplus}{D}$ learning spatial patterns

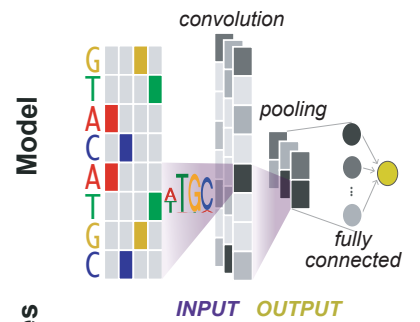

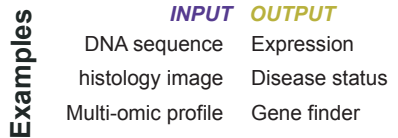

\section{Recurrent}

learning temporal or sequential patterns

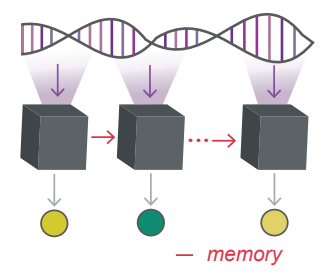

INPUT OUTPUT

DNA sequence TF binding

expression time series gene network

environmental variable population size time series over time

\section{Adversarial}

learning to synthesize

new instances

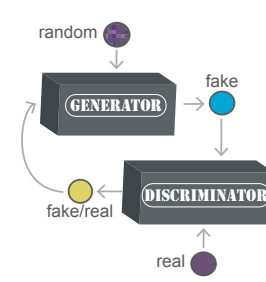

INPUT OUTPUT DNA sequences $\begin{aligned} & \text { Synthetic DNA } \\ & \text { sequences }\end{aligned}$

$\begin{aligned} \text { single-cell } & \text { Synthetic single-cell } \\ \text { expression pattern } & \text { expression pattern }\end{aligned}$ 